\title{
Evolution and Quality Analysis Algorithm of Consumer Online Reviews Based on Data Fusion and Multiobjective Optimization
}

\author{
Hu Wang, ${ }^{1}$ Tianbao Liang $\mathbb{D},{ }^{1,2}$ and Yanxia Cheng $\mathbb{D}^{1}$ \\ ${ }^{1}$ School of Management, Wuhan University of Technology, Wuhan, 430070 Hubei, China \\ ${ }^{2}$ School of Management, Zhongkai University of Agriculture and Engineering, Guangzhou, 510225 Guangdong, China \\ Correspondence should be addressed to Tianbao Liang; liangtb803@zhku.edu.cn
}

Received 10 August 2021; Revised 17 September 2021; Accepted 15 October 2021; Published 2 November 2021

Academic Editor: Mu Zhou

Copyright (C) $2021 \mathrm{Hu}$ Wang et al. This is an open access article distributed under the Creative Commons Attribution License, which permits unrestricted use, distribution, and reproduction in any medium, provided the original work is properly cited.

\begin{abstract}
With the rise of network strategies, various businesses using the Internet as a platform have been vigorously developed, among which the scale of e-commerce transactions has increased on a large scale. In order to deeply explore the role and advantages of data fusion and multiobjective optimization technology in consumer online reviews, this paper uses the new and old evaluation model comparison method, algorithm design method, and multiobject research method to collect samples, analyze the technical model, and streamline the algorithm. And it will create an analysis algorithm model that can improve and optimize the consumer's current online reviews. First, we choose the electricity supplier on the platform of a total of four mobile phones grabbed 32,145 comments. Based on this research on the number of online comment fields of consumers, the results show that $78 \%$ of the comments are less than 55 words, indicating that most of the online comments left by consumers are short comments; at the same time, a small number of consumers have left detailed comments. Description, the longest of is reached 612 words. On this basis, further study the efficiency and function analysis of the algorithm proposed in this paper, and we can see that DCDG-MOMA is used in 14-7 and 28-7 use cases as 1 and 2, respectively, which is the least, and at 40-7 and 50-7, the time used is 15 and 20 which is close to PBI, but it is also much less time than the MOMAD algorithm. This further shows that the algorithm really plays an effective role in the actual decision-making process. It has basically realized a more efficient algorithm for consumer online reviews under the background of applying data fusion and multiobjective optimization technology.
\end{abstract}

\section{Introduction}

In the past few decades, due to the rapid development of computer technology, each network platform to get a good leveraging some of scalable online business also has a corresponding increase. Among them, online shopping has become an important driving force for the development of e-commerce in our country. Online purchasing platform is where all is not well, that is, the problem undetected online purchasing presence. Based on this, this approach to reduce the information asymmetry mechanism of both parties to the transaction has become the first choice of major e-commerce platforms, and online reviews are currently widely adopted by various online shopping platforms. Online comment is one that can be evaluated for their customers to buy the product after shopping on the web electronic business plat- form and buy the product for consumers to purchase the follow-up to provide reference. However, with the rapid development of the Internet, this approach has also exposed its own bottlenecks, requiring the integration of new technologies for optimization.

Multiobjective optimization, as a research direction in multicriteria decision-making, refers to a multiobjective optimization problem in which the number of objects to be optimized is more than one, and the optimization should be carried out at the same time. When the variable domain of the problem is a finite set, we call this type of problem a multiobjective combinatorial optimization problem. Multiobjective optimization problems exist in a large number in the real world and have important research significance. How to design corresponding algorithms to solve such problems has also attracted more and more attention from 
scholars. This paper further proposes a multimodal sensor data fusion algorithm based on adaptive feedback adjustment. This method has good generalization ability, can more accurately estimate the mobile phone displacement, and dynamically adjust the screen display position, so that the mobile phone screen content is displayed smoothly and reduces frequent focus of the eyes, to achieve the effect of soothing visual fatigue. At the same time, it has great significance for online reviews and quality analysis.

Consumers have also been greatly facilitated in the development of science and technology, especially the combination of online reviews with data fusion and multiobjective optimization technology has made this advantage more obvious. Many scholars have done research here. In 2020, Jain and Vaidya reviewed most of the works reported by various authors about the use of social media during terrorist attacks in the past 10 years, how to conduct postattack social media analysis, and how to use social media analysis to detect terrorism, unrest, and hate behavior. For this goal, they used this review as a future antiterrorism research. However, this research path did not use technology [1]. In 2021, Lee and Hong used an online survey to collect data on 560 Amazon Mechanical Turk users. The results show that both perceived time pressure and perceived purchase uncertainty are positively correlated with the heuristic processing of online reviews, but negatively correlated with the systematic processing of online reviews. Their research finally discussed practical and academic significance, as well as future research directions. Although the research is complete, it lacks data support [2]. In 2016, Balakrishnan et al. conducted a bibliometric analysis based on more than 20,000 articles from Web of Science to study how knowledge about the two important multienergy systems of microgrids and smart grids has developed. Their findings identify areas that have been underresearched so far, provide a method for data fusion between different multienergy systems, and provide practical guidance for the implementation of multienergy system data fusion. Practicality is guaranteed, but the theory is somewhat inadequate [3]. In 2020, Park et al. introduced the publication of the second edition of Integrated Hierarchical Classification (ILC2), a free-side knowledge organization system (KOS), and reviewed the main changes introduced compared to the first edition (ILC1). The changes introduced in ILC2 include the following: the names and order of some major categories and the development of subcategories of various phenomena. The system can be accessed online for free via a PHP browser and SKOS format. However, there are deviations in the research process [4]. In 2019, Arora proposed that Industry 4.0 is the fourth industrial evolution: "The interconnection of the value creation process transcends the boundaries of a single company." To achieve this, there must be strong Internet access. Integration should not stay at the company boundary, but should also involve customers and serve as a digital business partner in Industry 4.0. It is a pity that it can only act on the industrial field and limit its development [5]. In 2016, Brill focused on theoretical issues, current developments, and emerging trends in all areas of the field, including detailed reviews of relevant recent literature. The editorial board and advisory board are composed of scholars and practitioners in the fields of public international law and European law to ensure that the review fully reflects the interrelationship between current developments and the continued development of this important legal theory and practice field. But based on this research, he did not understand [6]. In 2016, Meza et al. proposed a multiobjective optimization strategy based on particle swarm behavior with rotation and linear motion. The combination of explorationutilization led to the proposed cognitive algorithm, which was tested by multiple multiobjective optimization functions. Through statistical analysis, the proposed algorithm is compared with the standard particle swarm optimization multiobjective algorithm. Although the research process is quite inspiring, it is a pity that there is no conclusion [7].

The innovations of this article are as follows: (1) this research provides new research methods and ideas for exploring the impact of online reviews and also helps companies refine the impact of online reviews, so as to take targeted marketing measures; (2) how the price (favorable and bad comments) of online comments integrated by the new method acts on the evolution of consumer attitude is closer to reality and has stronger application value; (3) in terms of basic data acquisition, this article uses online comments serve as sample data. Compared with the traditional method, the analysis conclusion will be more scientific and effective. Through the above work, the application of theories based on data fusion and multiobjective optimization is more reasonable, and the model designed from this can better have a nationwide guiding application for consumer online reviews and quality analysis.

\section{Implementation Method of Online Comment and Analysis Algorithm Research Based on Data Fusion and Multiobjective Optimization}

2.1. Consumer Online Reviews. With the rise of the Internet, the spread of word-of-mouth is no longer limited to the real world but extends to the virtual Internet world. Virtual reality technology is a brand-new practical technology developed in the 20th century. Virtual reality technology includes computers, electronic information, and simulation technology, and its basic realization is that a computer simulates a virtual environment to give people a sense of environmental immersion. For example, foreign shopping platforms such as Amazon and e-Bay have launched product review functions as early as the 1990s, which has also aroused scholars' attention to the impact of online word-of-mouth. Online reviews, also known as online consumer reviews, are just one of the main manifestations of Internet word-of-mouth [8].

As a kind of online word-of-mouth, online reviews are not only related to but different from traditional offline word-of-mouth. From the perspective of connotation definition, online reviews and traditional offline word-of-mouth are both informal and noncommercial evaluation information about products or services [9], but they are not just simple common concepts. The connections and differences 
between the two are discussed below. The four common characteristics of online reviews and traditional offline word-of-mouth are as follows [10]:

\section{(1) High reliability}

Traditional offline word-of-mouth communication is generally carried out between relatives and friends, so recipients generally trust the source of information; online reviews are reviews left by consumers about products, because reviewers have no direct economic relationship with the merchants. The introduction of merchants is more objective and credible [11].

\section{(2) Two-way communication}

Different from one-way publicity methods such as advertising, word-of-mouth communication is two-way, and further interactive communication can be carried out between the communicator and the receiver, which can also be achieved by online reviews [12].

\section{(3) Less interference}

Compared with other communication methods, wordof-mouth information is less interfered by external factors due to word of mouth; online comments are generally kept on the Internet for more than one year, and the degree of interference is less [13].

\section{(4) Uncontrollable}

Online reviews are the same as traditional offline wordof-mouth communication. They are both intangible and intangible. Therefore, it is difficult for companies to control their communication. Moreover, in different industries, consumers have different dissemination intentions, which will affect potential consumer growth rate.

The difference between online reviews and traditional offline word-of-mouth is also very obvious. There are three differences:

\section{(1) Anonymity}

Because online comments publish information on the Internet, they can express their opinions in the most truthful manner without violating the law without worrying about any stakes [14].

\section{(2) Not limited by time and space}

Different from traditional word-of-mouth, online reviews can exist on shopping platforms for a long time and can be seen by anyone at any place and at any time, thus breaking the time and space limitations of word-of-mouth communication [15].

\section{(3) Content format}

Traditional offline word-of-mouth communication is mainly spread through language, facial expressions, body movements, etc., while online reviews are mainly spread through digital information such as text, pictures, and ratings [16].

Digital information dissemination technology includes theories, methods, technologies, and systems related to the acquisition, processing, storage, dissemination, management, security, and output of digital media information. It includes various information technologies such as computer technology, communication technology, and information processing technology. Comprehensive application technology, online comment is a process of information dissemination from initiator to receiver, as shown in Figure 1. The initiator of the comment publishes the content of the comment on the Internet and is searched or received by the receiver who needs the information. This is the communication process of online comments.

As can be seen from Figure 1, the initiator of online reviews directly determines the origin of online reviews and is also the basis for the spread of online reviews, which will also directly affect subsequent consumers' perceptions and behaviors of products [17]. Intelligent perception technology can be divided into four categories according to different objects and goals: perception technology based on human body analysis, perception technology based on vehicle analysis, perception technology based on behavior analysis, and perception technology based on image analysis. At the same time, theoretical combing is also critical. In view of the existing analysis, this article summarizes the relevant research on online reviews, as shown in Figure 2.

Judging from Figure 2, this paper analyzes several elements of online review research, the initiator (motivation, professionalism, etc.), the recipient (professionalism, network experience, etc.), the information processing process (ELM and HSM), and the dimensions of online comments themselves (quantity, valence, etc.) [18].

\subsection{Data Fusion and Multiobjective Optimization Technology.} Multimodal data fusion refers to the comprehensive use of computer technology to process multimodal data, so as to more accurately perceive information such as the state of the objective object and the environment in which it is located. Data fusion technology refers to information processing technology that uses computers to automatically analyze and synthesize several observation information obtained in time series under certain criteria to complete the required decision-making and evaluation tasks. Data fusion technology includes the collection, transmission, synthesis, filtering, correlation, and synthesis of useful information from various information sources to assist people in situation/environmental judgment, planning, detection, verification, and diagnosis. The focus of multimodal data fusion research includes various modal data feature extraction methods and multimodal data fusion algorithms. The focus of the fusion algorithm is to coordinate and complement different modal data and improve the accuracy of decision-making for uncertain data. The data fusion center fuses information from multiple sensors and can also fuse information from multiple sensors and the observation facts of the human-machine interface (this fusion is usually a decision-level fusion). 


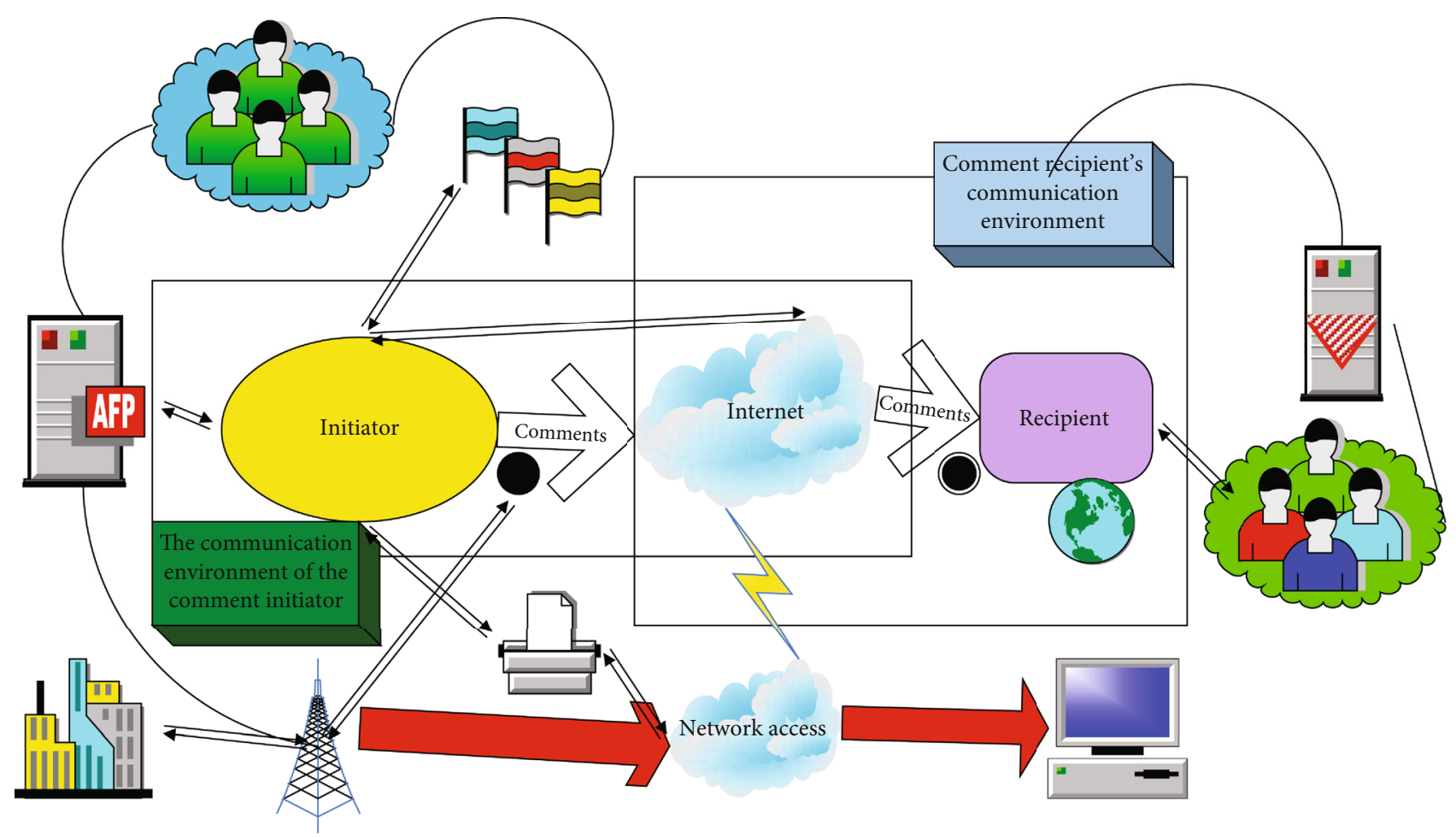

FIgURE 1: Online comment exchange process.

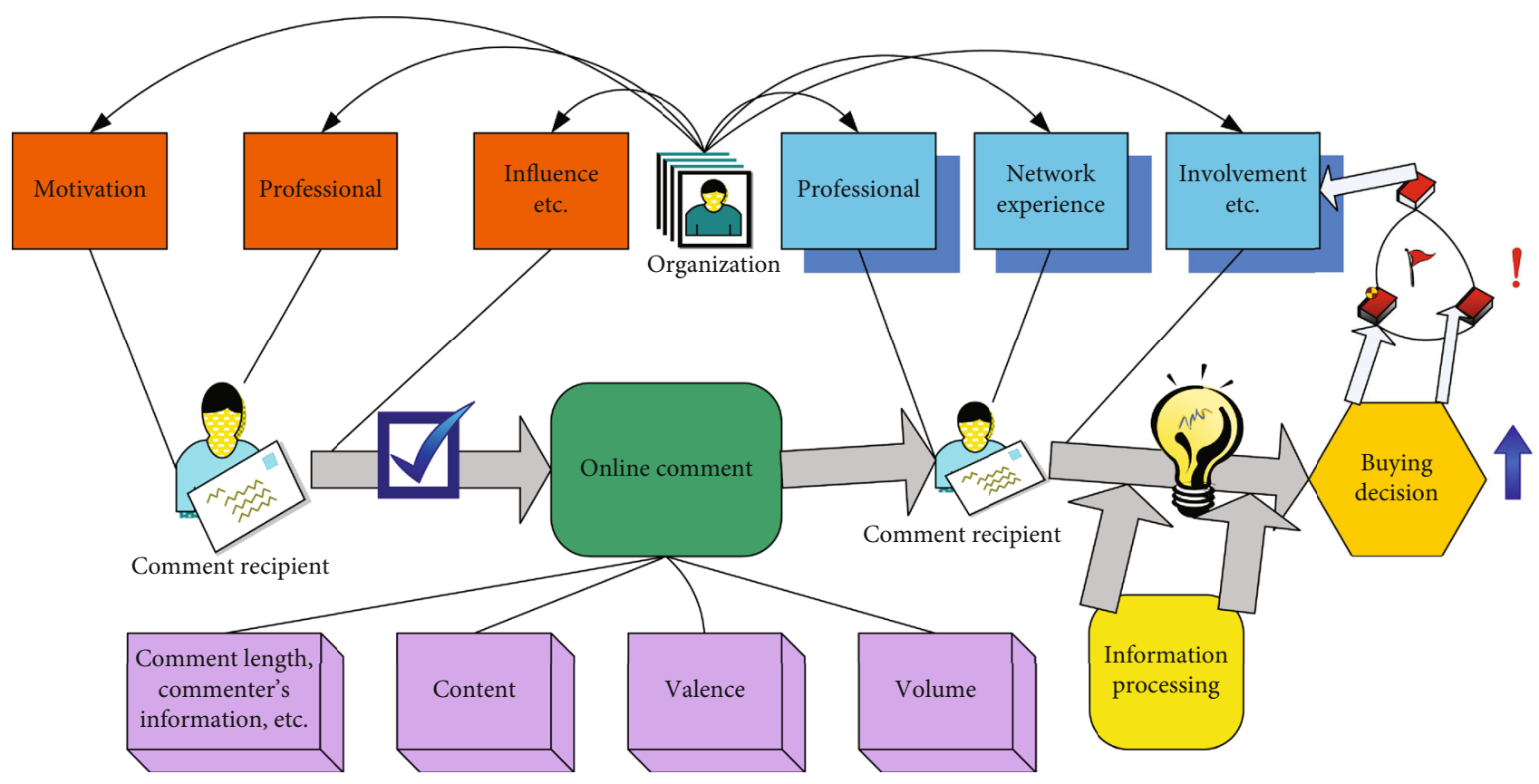

FIgURE 2: Theoretical research on online reviews.

Extract the symptom information, under the action of the inference engine. Match the symptoms with the knowledge in the knowledge base, make fault diagnosis decisions, and provide them to users. Compared with single mode, multimode system has the following advantages:

(1) Improve system stability
(2) Improve the continuity of information

Nowadays, multiobjective optimization problems are more and more widely used, involving many fields. In daily life and engineering, more than one index is often required to be optimized, and multiple indexes are often required to be optimized at the same time. A large number of problems 
can be attributed to a category of achieving multiple goals at the same time under certain constraints.

Multisensor data fusion is shown in Figure 3 according to the level of fusion: according to the different levels of fusion, it is divided into data layer fusion, feature layer fusion, and decision layer fusion [19].

As shown in Figure 3, multisensor data fusion can be divided into the following:

(1) Data Layer Fusion. The data detected by all sensors are fused, and the next step is performed from the fused data. This fusion method requires the sensors to detect the same type of data without losing the original data. The accuracy is high [20].

(2) Feature Layer Fusion. The data detected by each sensor is extracted separately, and then, these features are fused. This method compresses the original data to a certain extent, reduces the time required for subsequent processing, and improves the system's performance real-time.

(3) Decision-Making Layer Fusion. After each sensor detects the data, it performs feature extraction and target recognition and then fuses each recognition result. This method is the most inaccurate, because the information of each sensor is a high degree of concentration.

"Video networking" technology is the current advanced real-time high-definition video exchange technology, which can realize large-scale, high-quality, real-time, two-way, and symmetrical high-definition video full exchange. At present, there is no universal fusion method for multisensor data fusion, and it generally needs to be determined according to the specific application background. Weighted average method and neural network method are two classic data fusion methods based on measurement signals [21].

The weighted average method is the simplest and most intuitive method for processing information and data fusion. The basic process is as follows:

Assuming that $o$ sensors are used to measure a certain physical quantity, the output data of the $u$-th sensor is $a_{u}(u$ $=1,2, \cdots o$ ) and the weighting coefficient is $d_{u}$, and then, the output value of each sensor is weighted and averaged, and the weighted average fusion result is obtained as follows:

$$
\bar{a}=\sum_{u=1}^{o} d_{u} a_{u}+\sum_{u=2}^{k} d a_{c} .
$$

The weighted average method is the weighted average of the redundant information from each sensor, and the result is used as the fusion value. When applying this method, the system and the sensors used must be analyzed in detail to obtain the correct weights $\bar{a}$.

The biggest difference between the multiobjective optimization algorithm and the single-objective evolutionary algorithm in the calculation process is the individual fitness evaluation strategy. The use of a good individual fitness eval- uation strategy and selection strategy has a great effect on the performance of the evolutionary algorithm [22].

As an improvement of the NSGA algorithm, NSGAII uses a fast nondominated sorting strategy to establish a nondominated set. The method of constructing the nondominated set is as follows: first, set two parameter variables $t_{q}$ and $o_{q}$ for all individuals in the group $q$, and put the set $t_{m}$ in the individuals of $o_{m}-u=0$ are stored in the $m$ set. When $l_{u}=\partial$, the above operations are performed in sequence until all individuals are stratified and assigned a grade number [23].

Use a mathematical formula to describe the crowdedness of the environment in which individuals in the group are located. Suppose the crowded distance of the individual $u$ is $w_{u}$, and the crowded distance of the nonboundary individ$\mathrm{ual} \mathrm{u}$ is calculated as follows:

$$
w_{u}=\frac{\sum_{k=1}^{n}\left|l_{k}(u-1)-l_{k}(u+1)\right| / l_{k}^{\min }-l_{k}^{\max }}{n} .
$$

Among them, $l_{k}(u-1)$ and $l_{k}(u+1)$ are the objective function values of $u-1$ and $u+1$ near the individual $u$ on the $k$-th target, $l_{k}^{\min }$ and $l_{k}^{\max }$ are the maximum and minimum values of the $k$-th objective function, and $n$ is the number of objective functions. Number, the $k$-th gene of the next generation was obtained by simulating binary crossover [24].

$$
\begin{aligned}
& b_{1}^{\prime}(k)=0.5\left[\left(1-\tau_{m}(k)\right) a_{i}^{\prime}(k)+\left(1+\tau_{m}(k)\right) a_{1}^{\prime}(k)\right] \text {, } \\
& b_{2}^{\prime}(k)=0.5\left[\left(1-\tau_{m}(k)\right) a_{i}^{\prime}(k)+\left(1+\tau_{m}(k)\right) a_{2}^{\prime}(k)\right], \\
& \tau_{m}(k)=\left\{\begin{array}{ll}
(2 \rho(k))^{1 / o_{z}+1} \pi(k)<0.5, & \\
\left(\frac{1}{2(1-\pi(k))}\right)^{1 / o_{z}+1} & \text { anyother. }
\end{array} \pi(k) \in[0.1],\right.
\end{aligned}
$$

Polynomial transformation node can prevent the population due to conversion into a building partially optimized mode. Let $a_{u}^{s}(k)$ be the $k$-th gene of the $u$-th generation of individuals, and $a_{u}^{o}(k)$ and $a_{u}^{\prime}(k)$ are the next and next generations of $a_{1}^{i}(k)$. Individual $a_{u}^{s}(k)$ mutates to produce offspring $b_{u}^{i}(k)$. Among them, $\rho_{n}$ is a custom parameter, adjusted according to the actual situation, called the variation distribution index [25].

$$
\begin{aligned}
b_{u}^{s}(k) & =a_{u}^{\prime}(k)+\left(a_{i}^{o}(k)-a_{i}^{\prime}(k)\right) \overline{\tau_{m}}(k), \\
\overline{\tau_{m}}(k) & =\left\{\begin{array}{ll}
2 \bar{\pi}(k)^{1 / \pi_{n}-1} & \bar{\pi}(k)<0.5, \\
1-[2(1-\bar{\pi}(k))]^{1 / \pi_{n}-1} & \text { another. }
\end{array} \overline{\bar{\pi}(k)<0.5,}\right.
\end{aligned}
$$

Among them, $\overline{\tau_{m}}(k)$ is the exponential growth value, and $\bar{\pi}(k)<0.5$ is the discrimination criterion.

The performance evaluation of multiobjective optimization algorithm mainly adopts two aspects: convergence of 


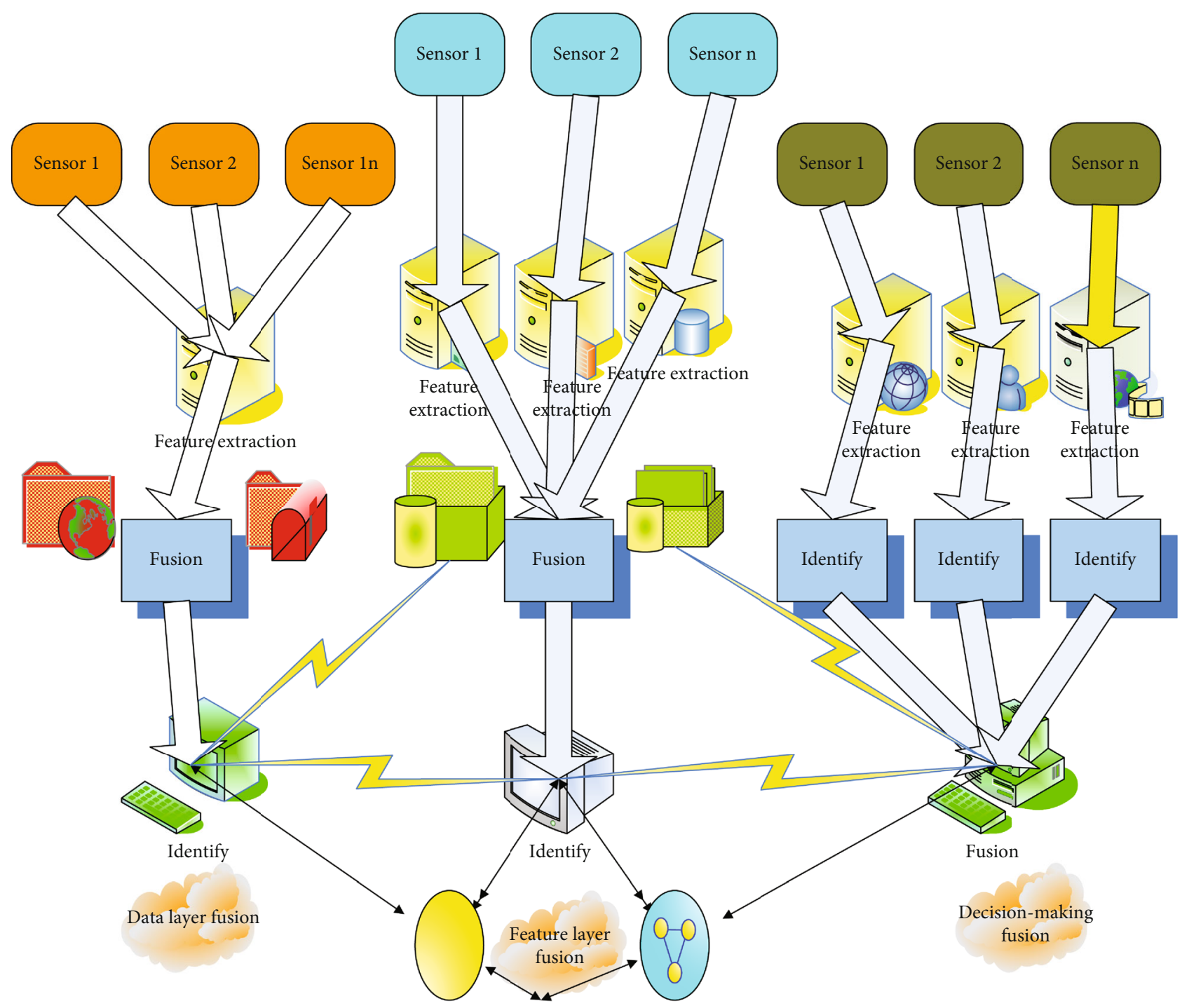

FIgURE 3: Schematic diagram of data fusion classification.

solution set and distribution breadth of solution set. In order to truly reflect the performance of the evolutionary algorithm, the following three evaluation indexes are mainly used in this paper, where the generation distance (GD) measures the algorithm convergence effect; the spatial distribution (SP) mainly evaluates the uniformity of the nondominant solution set distribution results, and the maximum spread cloth (MS) mainly analyzes the distribution universality of the nondominant solution set [26].

GD is used to evaluate the distance between the Pareto optimal front (PF) and the nondominated solution set front (PF) obtained by the algorithm in the ideal state of uniform distribution. The value is defined as follows:

$$
\mathrm{GD}=\sum_{u c}^{\exp } h_{k}+\frac{1}{o}\left(\sum_{u=1}^{o} w_{u}^{2}\right)^{1 / 2}
$$

For a specific optimization problem, $o$ is the number of individuals in $\mathrm{PF}$, and $w_{u}$ is the Euclidean distance between the $u$-th point in PF and the nearest point in PF in the target space. The uniformity of SP's main evaluation algorithm result set distribution is defined as follows:

$$
\mathrm{SP}=\frac{1}{\overline{w^{s}}}\left[\frac{1}{o} \sum_{u=1}^{o}\left(w^{\prime}-\bar{w}^{\prime}\right)^{2}\right]^{1 / 2}, \overline{w^{\prime}}=\frac{1}{o} \sum_{u=1}^{o} w_{u}^{s} .
$$

Among them, $w^{\prime}$ represents the Euclidean distance between the $u$-th individual in PF and its nearest, and $o$ is the number of individuals in PF [27]. MS is mainly used to measure the breadth of the distribution of nondominated solution sets obtained by evolutionary algorithms and is defined as follows:

$$
\mathrm{MS}=\sqrt{\frac{1}{n} \sum_{u=1}^{n}\left\{\frac{\max \left\{l_{u}^{\min }, l_{u}^{\min }\right\}-\min \left\{l_{u}^{\max }, l_{u}^{\max }\right\}}{l_{u}^{\min }-l_{u}^{\max }}\right\}}
$$


Among them, $n$ represents the number of targets, $l_{u}^{\min }$ and $l_{u}^{\max }$, respectively, represent the maximum and minimum values of the $u$-th dimension objective function in the $\mathrm{PF}$, and $l_{u}^{\mathrm{min}}$ and $l_{u}^{\mathrm{max}}$, respectively, represent the maximum and minimum values of the $i$-dimensional objective function in the PF.

\subsection{Algorithm}

2.3.1. Time Series Prediction Technology. Time series is a set of data points arranged in chronological order. Time series are very common in daily life. For example, the weekly average temperature of a certain place, the number of people dining in a week in a restaurant, and the stock price of a certain stock in one year can all be abstracted into time series for analysis. Time series forecasting technology is actually a regression forecasting method, which belongs to quantitative forecasting. Its basic principle is as follows: On the one hand, it recognizes the continuity of the development of things, uses past time series data for statistical analysis, and infers the development trend of things.

2.3.2. LSTM. LSTM is a variation of the cyclic neural network. Traditional RNN has the problem of disappearing gradients in terms of long-term dependence, which means that it will forget information that is far away from the time series. And LSTM gains the ability to learn long-term dependence by introducing three kinds of thresholds (forgetting threshold, input threshold, and output threshold) and has the ability to learn time series distance information. A simple LSTM network structure is shown in Figure 4.

As shown in Figure 4, the input is A, the output is G, and the information of the neural network will be passed to the next state through the cyclic structure X. On this basis, Figure 5 describes the internal principles of LSTM in detail.

As shown in Figure 5, in the figure, $u$ represents the sigmoid activation function, with a value range of 0 to 1 . Now let us introduce the forgetting gate of LSTM. The forgetting gate receives the output $g_{s-1}$ at the previous moment and the current input $a_{s}$ and then passes through a sigmoid layer to choose to forget some information. If the sigmoid output is 0 , it will be completely forgotten; if the output is 1 , it will be completely reserved [28].

$$
l_{s}=\sigma\left(d_{l} *\left[g_{s-1}, a_{s}\right]+y_{l}\right)
$$

LSTM input gate: first, the last output $g_{s-1}$ and the current input $a_{s}$ will pass through the sigmoid layer and output $u_{s}$. $u_{s}$ represents the probability of updating the state. Then, the last output $g_{s-1}$ and the current input $a_{s}$ will pass through the tanh layer to output the candidate new state $\widetilde{z_{s}}$.

$$
\begin{aligned}
& u_{s}=\sigma\left(d_{u} *\left[g_{s-1}, a_{s}\right]+y_{u}\right), \\
& \widetilde{z_{s}}=\cos g\left(d_{z} *\left[g_{s-1}, a_{s}\right]+y_{z}\right) .
\end{aligned}
$$

Subsequently, a new state $z_{s}$ needs to be generated, which is calculated from the output $l_{s}$ of the forget gate, the candidate new state $\widetilde{z}_{s}$ of the input gate, and the update probability $u_{s}$.

$$
z_{s}=l_{s} * z_{s-1}+u_{s} * z_{s}+\sqrt[d]{z_{d}^{s}}
$$

Output gate: the last output $g_{s-1}$ and the current input $a_{s}$ will pass through the sigmoid layer, output $p_{s}$, and determine which new state information will be output. Then, the new state $z_{s}$ is processed and multiplied by $p_{s}$ to determine the information $g_{s}$ output by the new state.

$$
\begin{aligned}
& p_{s}=\sigma\left(d_{p} *\left[g_{s-1}, a_{s}\right]+y_{p}\right)+\left(d_{p} *\left[g_{s-1}, a_{s}\right]+y_{p}\right) \\
& g_{s}=p_{s} * \sin g\left(z_{s}+\coprod_{z}^{u=1}(d c+o n)\right) .
\end{aligned}
$$

2.3.3. Linear Regression. Regression is a predictive modeling technique in which the estimated target variable is continuous [29]. The main forecasting technology models are linear regression model, moving average model, exponential smoothing model, trend extrapolation model, ARIMA forecasting model, Markov forecasting model, input-output forecasting model, grey forecasting model, artificial neural network forecasting model, etc. In reality, there are many applications of regression technology, such as forecasting stock market index, forecasting rainfall, road traffic, etc. Linear regression is divided into simple linear regression and multiple linear regressions. A variable to estimate the target variable is a simple linear regression (one-variable linear regression). For example, a data set $w=\left\{\left(a_{u}, b_{u}\right) / u=1,2,3\right.$ $\cdots o\}$ of $N$ observation data is collected, and a linear model is used to fit the observation data.

$$
l(a)=d_{1} a+d_{o}+\prod_{g=1}^{0} x_{g} .
$$

Among them, $l(a)$ is the data observation value, such that

$$
\max \sum_{u=1}^{o}\left[b_{u}-l(a)\right] b^{2} .
$$

Among them, $d_{1}$ and $d_{o}$ are the regression coefficients of simple linear regression. This method is also called the least square method. It tries to minimize the error between the estimated target variable and the real target variable through the appropriate regression coefficients $d_{1}$ and $d_{0}$.

Finding the regression coefficient $d_{0} d_{1}, \cdots d_{h}$ makes the following:

$$
\begin{aligned}
& \widehat{b}=d_{0}+d_{1} a_{1}+d_{2} a_{2}+\cdots+d_{h} a_{h}, \\
& \max \sum_{u=1}^{o}\left[b_{u}-\widehat{b}\right]+\left(\begin{array}{ll}
a & c \\
b & d
\end{array}\right)=u k .
\end{aligned}
$$

Among them, $b_{u}-\widehat{b}$ is an equation from the beginning to the end, and the above algorithm is established. 


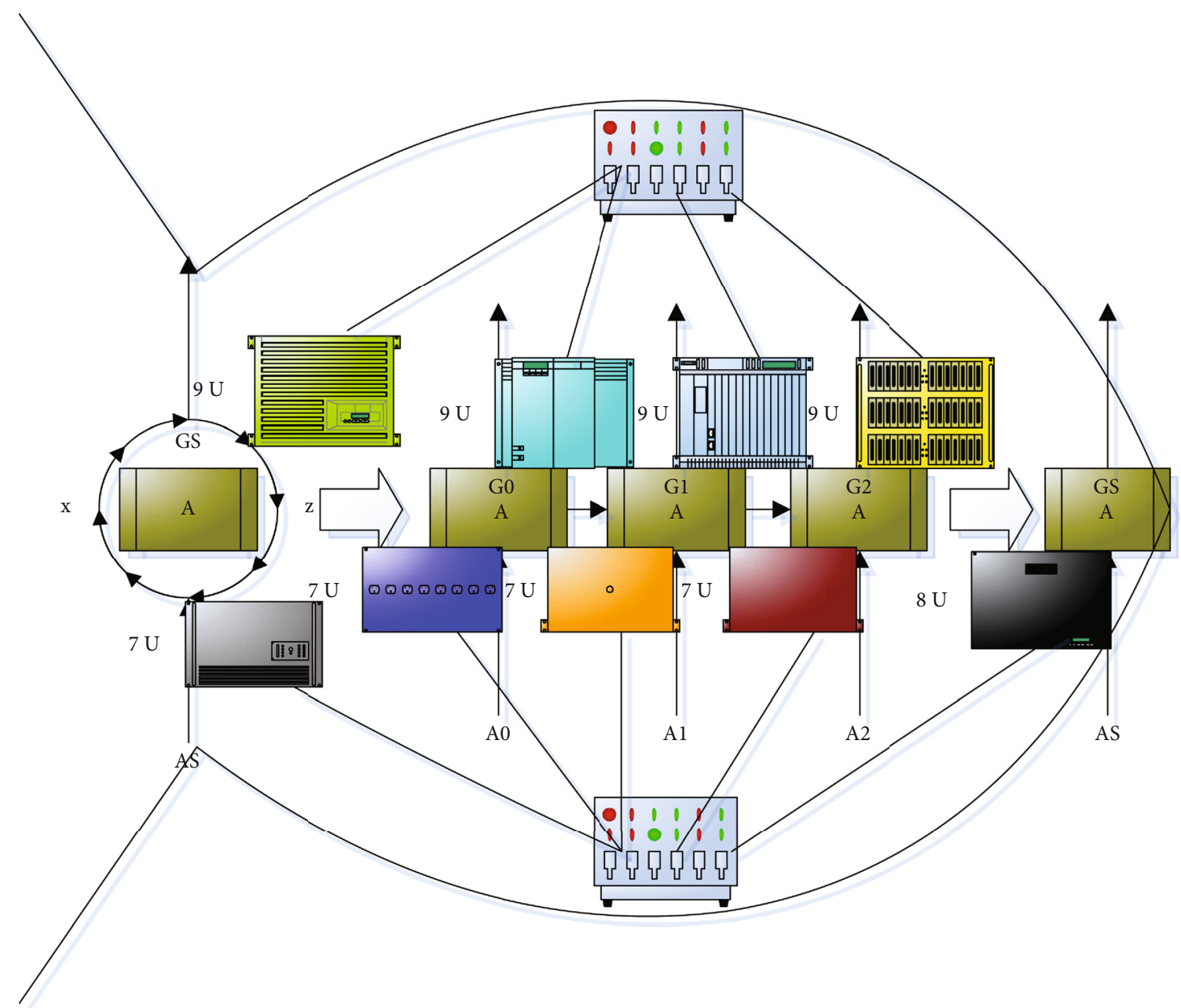

FIGURE 4: Schematic diagram of recurrent neural network LSTM.

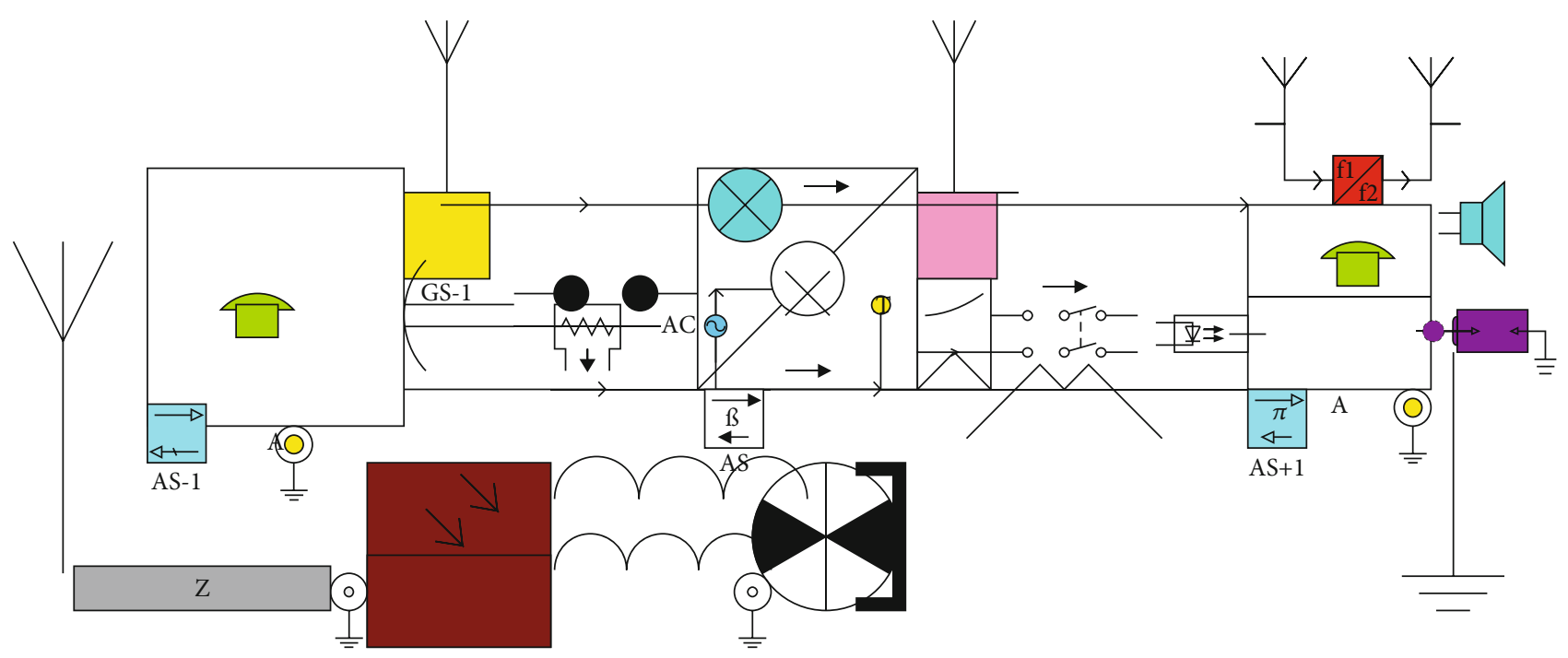

FIGURE 5: Schematic diagram of the LSTM structure of the recurrent neural network. 


\section{Based on Data Fusion and Multiobjective Optimization of Online Reviews and Analysis Algorithm Research Design and Implementation Method Experiments and Conclusions}

3.1. Experimental Setup. In order to verify the effectiveness of the algorithm MSIMOPSO proposed in this paper, three multiobjective optimization algorithms will be used as the comparison algorithm for this simulation experiment. These are the multiobjective evolutionary algorithm (MOEA/D) based on decomposition idea and the genetic algorithm based on fast nondominated sorting, NSGAII, and multistrategy improved multiobjective particle swarm optimization (MIMOPSO). And it adopts ZDT, DTLZ, and some UF test functions which are set as test functions. The parameter settings of each comparison algorithm and the corresponding population size and evaluation times of each test function are set as shown in Table 1.

Table 2 shows the setting method of the test function:

The above algorithms are run 30 times independently on the i5 processor, $8 \mathrm{G}$ RAM, and MATLAB2014a platform.

3.2. Model Simulation. In order to determine the value of each parameter in the model, this article obtained a series of data on a well-known domestic e-commerce website. According to the refined processing model (ELM), when consumers do not have time to carefully consider each piece of review information, their decision-making is usually quick and simple. This method usually only needs to extract the hidden meaning of the information to quickly make a choice, and the detailed description can be seen in the experiment, and the analysis can be seen below. Research shows that the number of reviews is one of the bases for many consumers to make quick decisions. In order to explore the influence of the number of reviews on consumer decisionmaking, this article collected reviews of these two categories of products from the mall using "laptop" and "flat-panel TV" as keywords, mainly including the number of reviews and praise rate.

This article first takes "laptop" as an example. A total of 785 products have been searched in the mall, of which 728 products have online reviews. Therefore, this article has collected a total of 258,473 online reviews of 728 products. Because some products under the same brand, although the models are different, there is little difference in configuration, and some products have less than 5 reviews, so the products can be reclassified. In order to calculate the information intensity of each product, this article classifies the 728 products collected by 30 brands, that is, each brand represents a product, and calculates the information intensity of each product based on this. Of course, the information intensity of each commodity can also be calculated separately, and the result does not affect the actual meaning of the information intensity calculation method. In the simulation of this article, each brand is regarded as a product, and reviews of products under the same brand are included in the reviews of that brand. The data is shown in Figure 6.
TABLE 1: Comparison algorithm parameter settings.

\begin{tabular}{lccc}
\hline $\begin{array}{l}\text { Algorithm } \\
\text { name }\end{array}$ & Parameter settings & $\begin{array}{c}\text { Group } \\
\text { name }\end{array}$ & Possibility \\
\hline MSI & $W 1=0.3, W 2=0.8, Z 1=$ & $\mathrm{A}$ & $95.2 \%$ \\
NSG & $\mathrm{PC}=0.8, \mathrm{PN}=\mathrm{O}$ & $\mathrm{B}$ & $96.4 \%$ \\
MOE & $T=0.1 N, \mathrm{ZE}=0.9$ & $\mathrm{C}$ & $91.8 \%$ \\
MIM & $W=0.108, Z 1=Z 2=1.88$ & $\mathrm{D}$ & $93.5 \%$ \\
\hline
\end{tabular}

TABLE 2: Test function settings.

\begin{tabular}{lccc}
\hline $\begin{array}{l}\text { Function } \\
\text { name }\end{array}$ & $\begin{array}{c}\text { Population } \\
\text { size }\end{array}$ & $\begin{array}{c}\text { External archive } \\
\text { size }\end{array}$ & $\begin{array}{c}\text { Evaluation } \\
\text { times }\end{array}$ \\
\hline ZDT & 90 & 90 & 30000 \\
DTLZ & 140 & 140 & 100000 \\
UF & 190 & 190 & 300000 \\
\hline
\end{tabular}

It can be seen from Figure 6 that under the support of the data parameters in the figure, the information intensity of each brand product is calculated by the calculation formula, and the results are shown in Table 3.

In order to determine the influence of information intensity on consumer acceptance-acceptance probability, this paper selected 3 products from 30 products for simulation, namely, THINK, $n=0.24$; Samsung, $n=0.054$; and Jumper, $n=0.00024$. The information intensity of these three commodities, respectively, represents the high information intensity, medium information intensity, and low information intensity of the online review information of these products.

3.3. Data Collection. This article uses the GooSeeker web crawler software developed based on Python to obtain online comment data on the four popular smartphones of domestic A mobile phone, domestic B mobile phone, foreign A mobile phone, and foreign $B$ mobile phone on the online shopping platform. This article crawls the user name, user rating, evaluation time, number of likes, number of replies, and details of the comment text of the comment. The comment interface and comment data acquisition methods of the other three mobile phones are similar, so I will not repeat them here.

As shown in Table 4, there are a total of 32,145 comments on the four mobile phones crawled in this paper: domestic A mobile phone, domestic B mobile phone, foreign A mobile phone, and foreign $B$ mobile phone. Among them, 15,487 comments were obtained from the $x$ platform and 16,658 comments were obtained from the $y$ platform. The number of comments on each mobile phone was basically the same, and the number of comments on foreign mobile phones was slightly larger. The time span of the comment is nearly 6 months, which is basically consistent with the current life cycle of mobile phones.

Then, study the number of words used in their comments, as shown in Figure 7. 


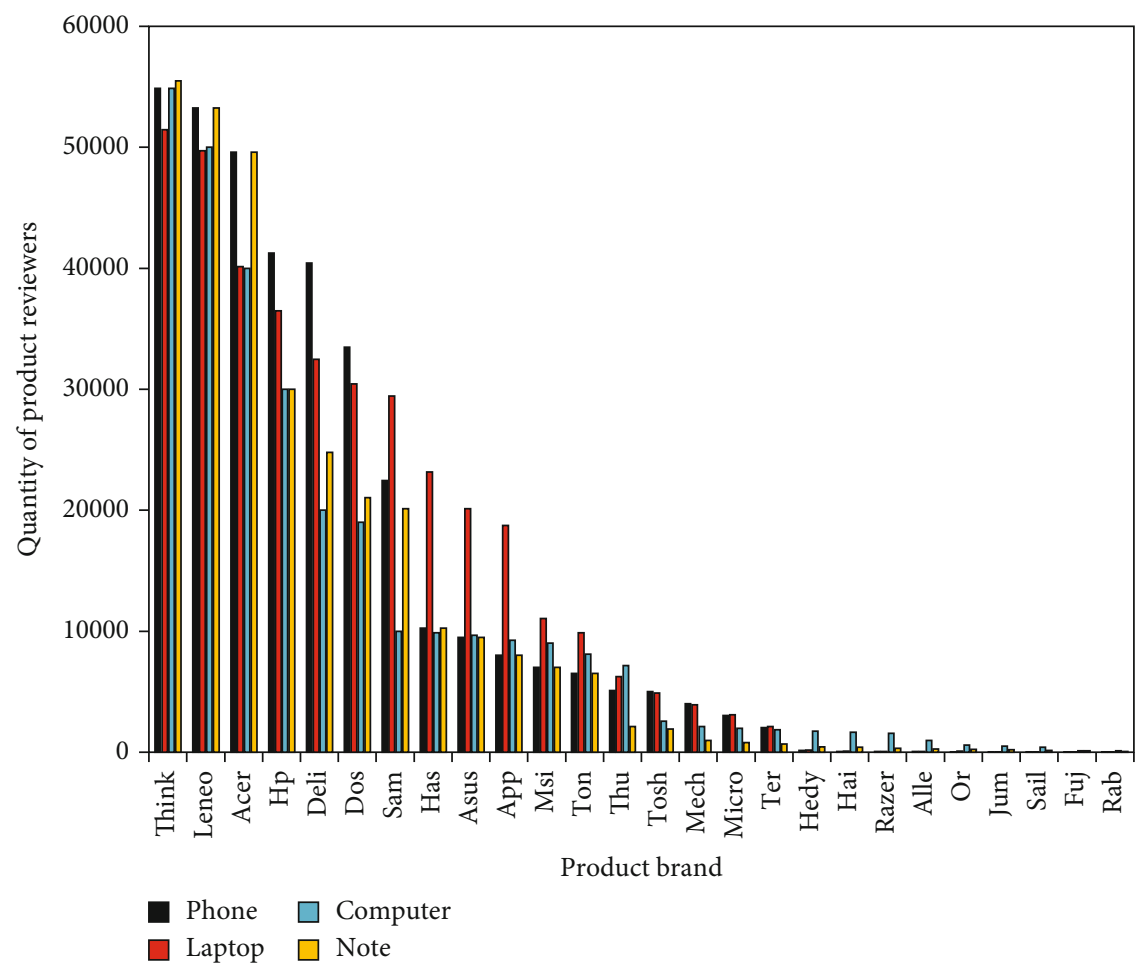

FIgURE 6: Number of reviews of each brand product.

TABLE 3: Information intensity of laptops of various brands.

\begin{tabular}{lccc}
\hline Brand & Information intensity & Brand & Information intensity \\
\hline THINK & 0.24 & ME & 0.005 \\
LENEO & 0.18 & MICRO & 0.005 \\
ACER & 0.08 & TER & 0.008 \\
HP & 0.07 & HEDY & 0.015 \\
DELI & 0.05 & HAI & 0.0015 \\
DOS & 0.04 & RAZER & 0.0014 \\
SAM & 0.054 & ALL & 0.00133 \\
\hline
\end{tabular}

It can be seen from Figure 7 that it is a word frequency chart based on the word count of each comment. This figure shows that $78 \%$ of reviews are less than 55 words, indicating that most of the online reviews left by consumers on shopping platforms are short reviews; at the same time, a small number of consumers have left a detailed description of their shopping experience. The longest comment has reached 612 words.

3.4. Survey Samples and Analysis. For all the algorithms, we compared the final results of the experiment. From these table data, we can clearly observe that RLG-PLS is superior to other algorithms in all MOKP and MOTSP test cases. This conclusion also shows that RLG-PLS has good convergence performance. The neighbor structure in the local search process is defined as follows: for a solution, one of its neighbors can be removed by removing the two hardware devices that have been placed, and then, the two hardware devices are randomly selected under the premise that all constraints are met. Choose two locations for placement, and a feasible configuration solution obtained after placement is a new neighbor. In this article, all algorithms use this neighbor structure definition method.

We can see from Table 5 that the value of DCDGMOMA on the c-metric index is significantly better than algorithms such as MOEA/D-LS and MOMAD. Therefore, we can get that the convergence performance of DCDGMOMA is better than these compared algorithms.

In order to further verify the pros and cons of the algorithms, we also calculated the hypervolume index of the solutions obtained by these algorithms, as shown in Figure 8.

As shown in Figure 8, the results of DCDG-MOMA on $\mathrm{IH}$ are better than the compared algorithms. According to the values of c-metric and IH, it can be seen that DCDG is significantly better than the compared algorithms, which conforms to the shortcomings of the decomposition methods analyzed in the article in maintaining diversity. To further study the time it takes for the algorithm to run, please see Figure 9.

It can be seen from Figure 9 the calculation time spent by all algorithms in running different DAP test cases. From these data, we can see that DCDG-MOMA uses 1 and 2 for 14-7 and 28-7 use cases, respectively. For the least, the time used on $40-7$ and $50-7$ is 15 and 20 is close to PBI, but it is also much less time than MOMAD algorithm.

3.5. Effect Analysis. For the device allocation problem in DIMA, the solution obtained by DCDG-MOMA and other algorithms is a solution set. In practice, decision makers 
TABLE 4: Statistical description.

\begin{tabular}{|c|c|c|c|c|c|}
\hline Mobile phone brands & Time to market & $x$ platform & $y$ platform & Total & Comment time \\
\hline Domestic A mobile phone & 2016.3.1 & 3000 & 3600 & 6600 & \multirow{5}{*}{ 2015.9-2016.10 } \\
\hline Domestic B mobile phone & 2015.12 .9 & 2000 & 2400 & 4400 & \\
\hline Foreign A mobile phone & 2015.9 .9 & 5487 & 6658 & 12145 & \\
\hline Foreign B mobile phone & 2016.8 .3 & 5000 & 4000 & 9000 & \\
\hline Number of comments & & 15,487 & 16,658 & 32,145 & \\
\hline
\end{tabular}
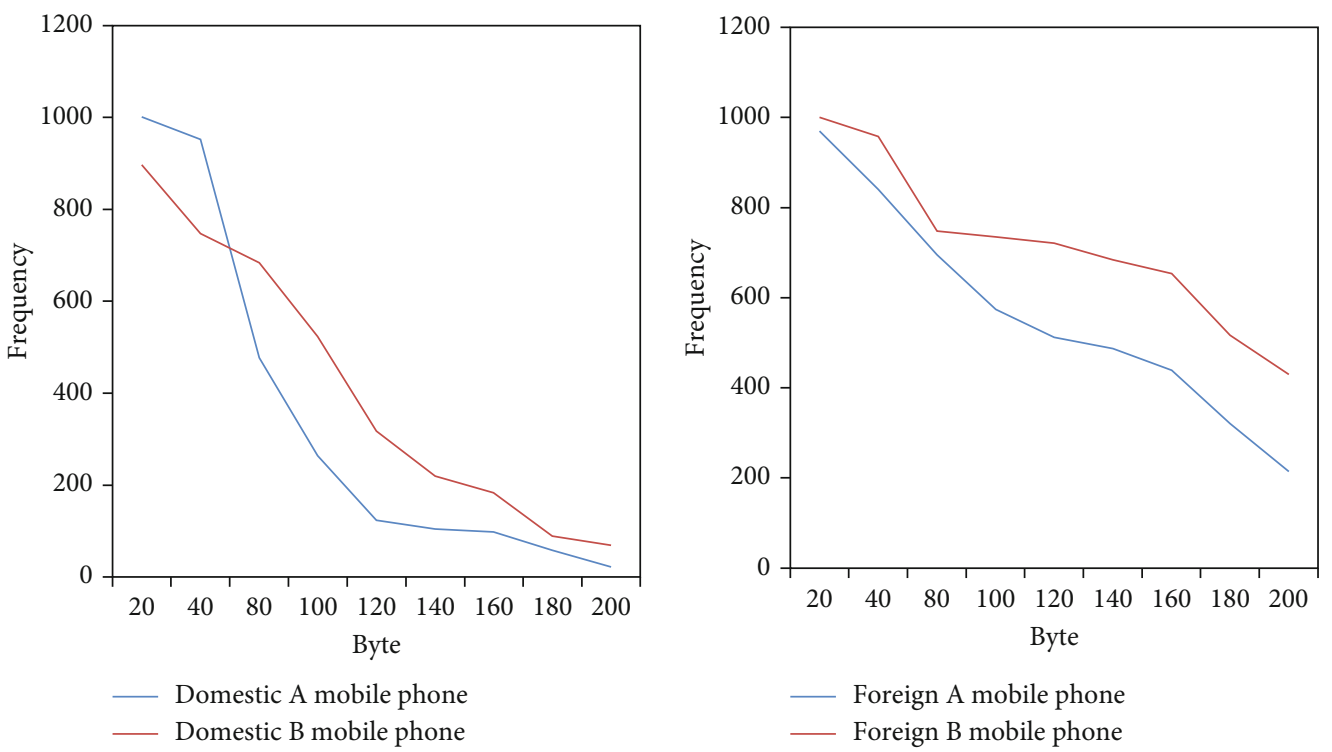

FIGURE 7: Frequency statistics of online comments.

TABLE 5: c-metric (\%) of DCDG-MOMA, MOEA/D-LS, and MOMAD on DAP.

\begin{tabular}{lcccccccc}
\hline \multirow{2}{*}{ Test case } & MOEA/D & WS & MOEA/D & TCH & MOEA/D & \multicolumn{2}{c}{ PBI } & \multicolumn{2}{c}{ MOMAD } \\
& $Z(X, Y)$ & $Z(Y, X)$ & $Z(X, Y)$ & $Z(Y, X)$ & $Z(X, Y)$ & $Z(Y, X)$ & $Z(X, Y)$ & $Z(Y, X)$ \\
\hline $13-7$ & 70.1 & 0.28 & 67.89 & 2.98 & 69.8 & 0.11 & 77.58 \\
$22-7$ & 6.12 & 4.3 & 18.56 & 3.98 & 28.56 & 3.6 & 9.11 \\
$42-7$ & 17.25 & 18.8 & 44.85 & 40.21 & 48.25 & 18.25 & 44.12 \\
$48-7$ & 44.25 & 9.96 & 44.36 & 9.58 & 55.36 & 9.9 & 22.11 \\
\hline
\end{tabular}

often choose one of these solutions as the final implementation plan without any preference. In this article, EMU will be used to determine the solution of interest, and the selected solution will be compared with the solution generated by the greedy strategy, and the practical application of DCDGMOMA will be analyzed. As shown in Figure 10, for the two use cases 14-7 and 28-7, 25 independent runs, the $\mathrm{IH}$ is the median solution using EMU.

As shown in Figure 10, the target value (mass, OIC) corresponding to the selected solution and the target value corresponding to the three solutions generated by the greedy strategy are displayed. According to these target values, the solution obtained by DCDG-MOMA is much better than the solution generated by the greedy random method, especially in the 28-7 example, which further shows that DCDG-
MOMA does play a role in the actual decision-making process.

\section{Discussion}

By using a number of comments in the above survey of online reviews, we discuss the key factors influencing changes in consumer experience a sense of online reviews. I am here with two complete description of direct review. The research results show that the information intensity of online reviews has a very important impact on consumers' receiving experience. Specifically, they are less affected by online reviews. In terms of experience changes, they have a higher probability of receiving review information, but a lower probability of accepting the information. This has 


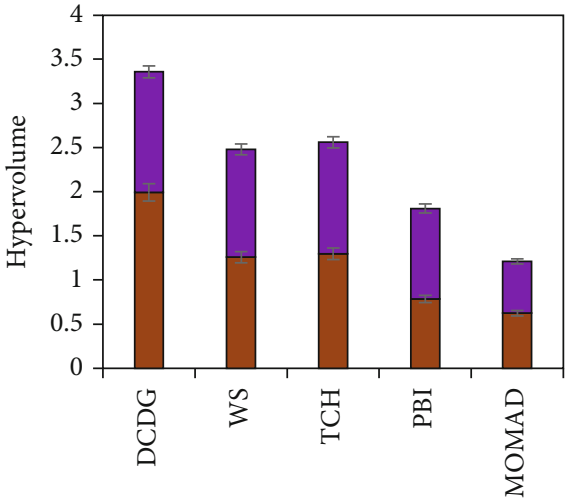

Algorithms

28 to 7

- 14 to 7

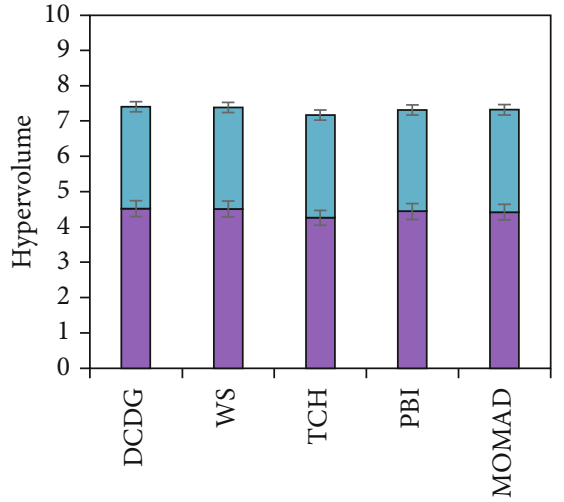

Algorithms

$\square 40$ to 7

50 to 7

Figure 8: DCDG-MOMA, MOEA/D-LS, and MOMAD overvolume index results of 20 runs on all test cases.

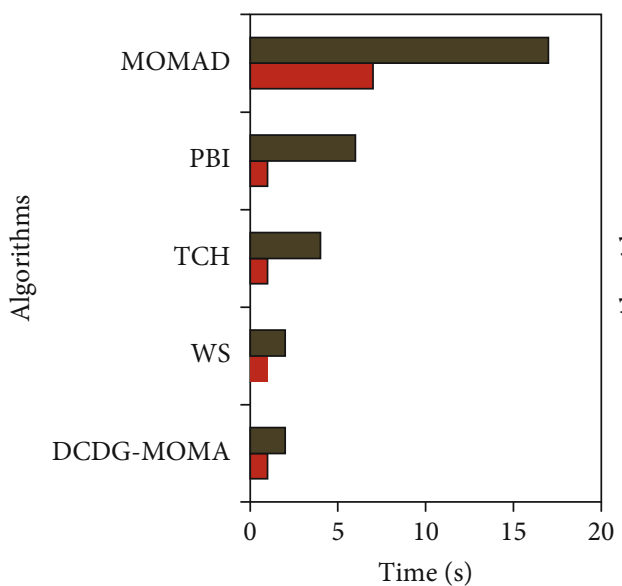

28 to 7

$\square 14$ to 7

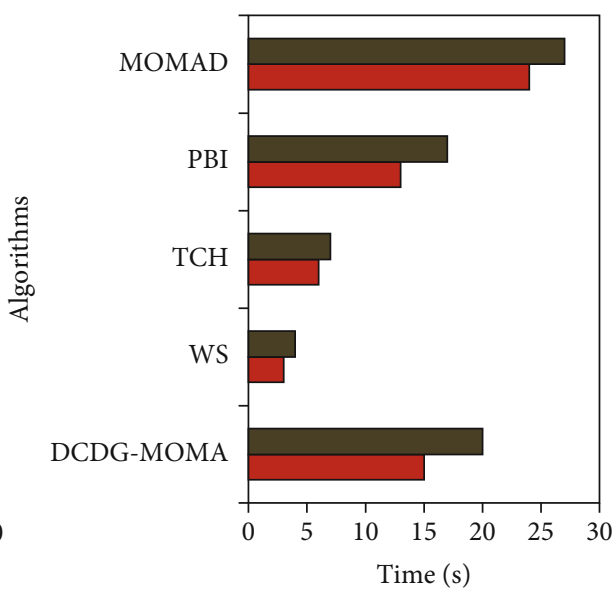

50 to 7

口 40 to 7

Figure 9: Running time of DCDG-MOMA, MOEA/D-LS, and MOMAD on different test cases.
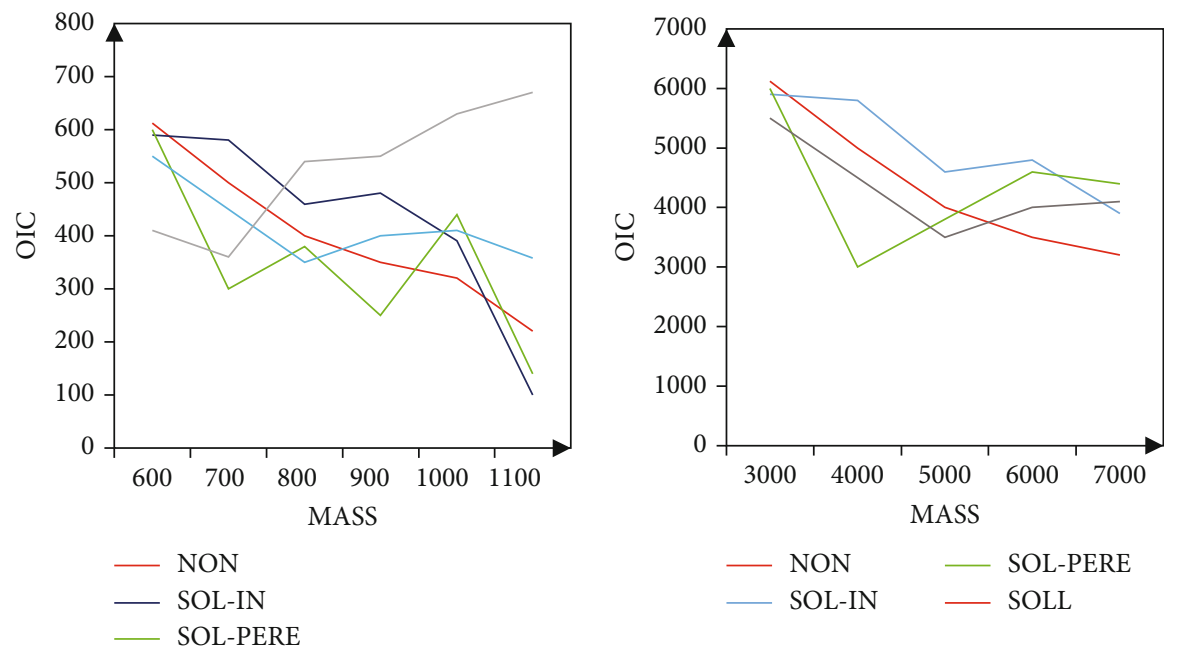

FIgURe 10: Diagram of the solution obtained by EMUr. 
similar conclusions to the previous study by Park et al. It can be seen that consumers are more inclined to use marginal information processing methods to process review information, that is, to grasp the key points of review information and make decisions quickly. It can be seen from the research results of this article that the number of online reviews is an important factor influencing consumer decision-making. At the same time, previous studies have found that consumers are more likely to form a corresponding impression of the product in the early stage and are basically no longer affected by online reviews in the later stage. The research results of this article found that individual consumers are more likely to receive relevant information but are basically unaffected during the acceptance stage, which further reflects the independence of their comments.

First of all, in view of the impact of online reviews on consumer attitudes, companies should take measures to vigorously promote the increase in the number of reviews, because a product with a large number of reviews will increase consumers' acceptance of review information, prompting them to pay more attention to the product. Only when the product attracts the attention of consumers can it further encourage consumers to have certain existing tendencies and encourage them to further accept the product. Second, enhance the guiding nature of online reviews. Consumers who use the new algorithm have a higher probability of receiving and accepting review information, which enhances the guidance of reviews, while presenting products, such as under the product picture, some product praises are displayed, so that consumers can closely compare reviews and products. Combining them will further deepen consumers' impression of the product and increase their likelihood of accepting the product.

\section{Conclusions}

With the rise of e-commerce, online communities, etc., online reviews have become an important area for academics and companies to discuss consumer behavior. This article uses a variety of scientific methods, such as parameter comparison method, data fusion method, and algorithm optimization method; the purpose is to collect data and design a reasonable algorithm model. The algorithm proposed in this paper has good efficiency and ability to serve consumers. The shortcomings of this article are as follows: first, the algorithm has excellent performance in solving two goals, but as the number of goals increases, the effectiveness of the solution selection part will be weakened even when the three goals are reached. Secondly, this algorithm works well in relatively regular network scenarios, but it is not obvious in other irregular scenarios, with insufficient generalization ability and insufficient flexibility. Therefore, in the next step of research, we need to increase the algorithm's segment points to make the algorithm more universal, to accept a wider range of goals, and to enhance its stability, and its efficiency will not change with environmental changes. In the end, realizing the algorithm model proposed in this paper can have wider applications.

\section{Data Availability}

The data that support the findings of this study are available from the corresponding author upon reasonable request.

\section{Conflicts of Interest}

The authors declared no potential conflicts of interest with respect to the research, authorship, and/or publication of this article.

\section{Acknowledgments}

This work was supported by the National Social Science Foundation of China (16BGL089).

\section{References}

[1] P. Jain and A. S. Vaidya, "Analysis of social media based on terrorism - a review," Vietnam Journal of Computer Science, vol. 8, no. 1, pp. 1-21, 2020.

[2] J. Lee and I. B. Hong, "The influence of situational constraints on consumers' evaluation and use of online reviews: a heuristic-systematic model perspective," Journal of Theoretical and Applied Electronic Commerce Research, vol. 16, no. 5, pp. 1517-1536, 2021.

[3] D. Balakrishnan, A. B. Haney, and J. Meuer, "What a MES(s)! A bibliometric analysis of the evolution of research on multienergy systems," Electrical Engineering, vol. 98, no. 4, pp. 16, 2016.

[4] Z. Park, C. Gnoli, and D. P. Morelli, "The second edition of the integrative levels classification: evolution of a KOS," Journal of Data and Information Science, vol. 5, no. 1, pp. 39-50, 2020.

[5] C. Arora, "Digital business leadership: digital transformation, business model innovation, agile organization, change management," Computing Reviews, vol. 60, no. 4, pp. 162-162, 2019.

[6] Brill, "Austrian review of international and European law," Austrian Review of International \& European Law Online, vol. 1, no. 1, pp. 121-143, 2016.

[7] J. Meza, H. Espitia, C. Montenegro, and R. G. Crespo, "Statistical analysis of a multi-objective optimization algorithm based on a model of particles with vorticity behavior," Soft Computing, vol. 20, no. 9, pp. 3521-3536, 2016.

[8] S. Otto, "Religion in science fiction: the evolution of an idea and the extinction of a genre, Steven Hrotic, Bloomsbury, 2014 (ISBN 978-1-4725-3355-5), ix + 225 pp., hb £65," Reviews in Religion \& Theology, vol. 23, no. 3, pp. 323-326, 2016.

[9] M. Zhou, Y. X. Long, W. P. Zhang et al., "Adaptive genetic algorithm-aided neural network with channel state information tensor decomposition for indoor localization," IEEE Transactions on Evolutionary Computation, vol. 25, 2021.

[10] H. Kaneko, Y. Otsuka, Y. Kubota, and G. Wakabayashi, "Evolution and revolution of laparoscopic liver resection in Japan," Annals of Gastroenterological Surgery, vol. 1, no. 1, pp. 33-43, 2017.

[11] G. C. Nunez-Mir, B. V. Iannone III, B. C. Pijanowski, N. Kong, and S. Fei, "Automated content analysis: addressing the big literature challenge in ecology and evolution," Methods in Ecology \& Evolution, vol. 7, no. 11, pp. 1262-1272, 2016. 
[12] D. Guido, H. B. Song, and S. Anke, Big Data Analytics for Cyber-Physical Systems: Machine Learning for the Internet of Things, Elsevier, 2019.

[13] B. T. P. Chern and F. B. S. Ahmad, "Supply chain evolution. A study of opportunities and challenges of virtual kitchens in Malaysia," Journal of Critical Reviews, vol. 7, no. 16, pp. 361368, 2020.

[14] F. Vigneron and P. V. Ca, "Evolution in the chemical making of gold oxidation catalysts," ChemInform, vol. 47, no. 19, pp. 192-198, 2016.

[15] M. Zhou, Y. M. Wang, Z. S. Tian, Y. H. Lian, Y. Wang, and B. Wang, "Calibrated data simplification for energy-efficient location sensing in internet of things," IEEE Internet of Things Journal, vol. 6, no. 4, pp. 6125-6133, 2019.

[16] D. C. Adams and M. L. Collyer, "Phylogenetic comparative methods and the evolution of multivariate phenotypes," Annual Review of Ecology Evolution and Systematics, vol. 50, no. 1, pp. 1-21, 2019.

[17] S. K. Kamalakarababu, A. Sam, and S. Varghese, "Clinical profile and short-term outcome of acute nephritic syndrome in children," Journal of Evolution of Medical and Dental Sciences, vol. 6, no. 88, pp. 6157-6160, 2017.

[18] Y. Hu, C. Hu, S. Fu et al., "Survey on popularity evolution analysis and prediction," Journal of Electronics \& Information Technology, vol. 39, no. 4, pp. 805-816, 2017.

[19] H. Fan, H. Zhan, S. Cheng, and B. Mi, "Research and application of multi-objective particle swarm optimization algorithm based on $\alpha$-stable distribution," Xibei Gongye Daxue Xuebao/Journal of Northwestern Polytechnical University, vol. 37, no. 2, pp. 232-241, 2019.

[20] Q. Zhou, C. Zhang, S. X. Zhao, and B. Chen, "Measuring book impact based on the multi-granularity online review mining," Scientometrics, vol. 107, no. 3, pp. 1435-1455, 2016.

[21] Y. Guo, P. Yan, D. Wu, H. Zhou, Y. Shi, and R. Yi, “Analysis method for factors influencing gear hobbing quality based on density peak clustering and improved multi-objective differential evolution algorithm," International Journal of Computer Integrated Manufacturing, vol. 34, no. 4, pp. 1-22, 2021.

[22] Y. Hou, H. G. Han, and J. Qiao, "Adaptive multi-objective differential evolution algorithm based on the dynamic parameters adjustment," Control and Decision, vol. 32, no. 11, pp. 1985-1990, 2017.

[23] M. Zhou, Y. X. Lin, N. Zhao, Q. Jiang, X. L. Yang, and Z. S. Tian, "Indoor WLAN intelligent target intrusion sensing using ray-aided generative adversarial network," IEEE Transactions on Emerging Topics in Computational Intelligence, vol. 4, no. 1 , pp. $61-73,2020$.

[24] L. N. Deng and X. F. Yao, "Research on the fusion algorithm of infrared and visible images based on non-subsampled shearlet transform," Tien Tzu Hsueh Pao/Acta Electronica Sinica, vol. 45, no. 12, pp. 2965-2970, 2017.

[25] Z. Hao, Z. Lou, and Y. Fan, "Study on the evolution mechanism of subsurface defects in nickel-based single crystal alloy during atomic and close-to-atomic scale cutting," Journal of Manufacturing Processes, vol. 68, no. 3, pp. 14-33, 2021.

[26] H. Xie, "Research and case analysis of apriori algorithm based on mining frequent item-sets," Open Journal of Social Sciences, vol. 9, no. 4, pp. 458-468, 2021.

[27] X. Xu, D. Cui, Y. Li, and Y. Xiao, "Research on ship trajectory extraction based on multi-attribute DBSCAN optimisation algorithm," Polish Maritime Research, vol. 28, no. 1, pp. 136148, 2021.

[28] C. You, Q. Li, W. Li, and B. Xu, "Research on healthy evolution and driving mechanism of cultivated land utilization system in Chongqing based on PSR-TOPSIS model," Advance in Agriculture Science, vol. 2, no. 4, pp. 149-165, 2020.

[29] H. Jia, Z. Wei, X. He, and M. Li, “A research on lane marking detection algorithm based on neural network and least squares method," Qiche Gongcheng/Automotive Engineering, vol. 40, no. 3, pp. 363-368, 2018. 